\title{
USO DEL DISEÑO FACTORIAL EN LOS ENSAYOS DE LIBERACIÓN CONTROLADA DEL ÁCIDO 1,3 INDOLACÉTICO CARGADO EN MATRICES DE QUITOSANO
}

\author{
Ana Valderrama Negrón ${ }^{a}$, Christian Jacinto Hernández ${ }^{a}$, \\ Susana Ponce García ${ }^{\mathrm{b}}$, Luis Manrique Pollera ${ }^{\mathrm{a}}$
}

\begin{abstract}
RESUMEN
En este trabajo se utilizó el diseño factorial de experimentos para la preparación de partículas de quitosano cargadas con la hormona vegetal ácido 1,3-indolacético (AIA) y posteriormente estudiar la liberación controlada en agua. Las partículas de quitosano cargadas se prepararon disolviendo la hormona y el quitosano en ácido acético haciéndose gotear luego en solución de $\mathrm{NaOH}$ con agitación constante. Se utilizó el Diseño Factorial $2^{3}$ cuyos factores a estudiar fueron: la concentración de quitosano (\% quitosano), tiempo de gelación en $\mathrm{NaOH}$ (tiempo en $\mathrm{NaOH})$ y concentración de tripolifosfato de sodio (TPP) (\% de TPP). Como respuesta se obtuvo la cantidad de hormona liberada en dos horas, medida espectrofotométricamente a $285 \mathrm{~nm}$. Los resultados del diseño experimental indicaron que los tres factores influyen en la cantidad liberada de AIA en dos horas, en donde a mayor concentración de quitosano aumenta la velocidad de liberación, mientras que a mayor concentración de TPP y mayor tiempo de gelación en $\mathrm{NaOH}$ disminuye la velocidad de liberación.
\end{abstract}

Palabras clave: Diseño factorial, liberación controlada, ácido 1,3 indolacético, quitosano.

\begin{abstract}
In this work we used the factorial design of experiments for the preparation of chitosan particles loaded with the vegetable hormone 1,3-indoleacetic acid (AIA) to study its controlled release in water. The chitosan particles were prepared by dissolving the hormone and chitosan in acetic acid and then dripping in $\mathrm{NaOH}$ solution with constant stirring. Factorial Design $2^{3}$ was used to study the concentration of chitosan (\% chitosan), gelation time in $\mathrm{NaOH}$ (time in $\mathrm{NaOH}$ ) and sodium tripolyphosphate (TPP) concentration (\% of TPP). In response, the amount of hormone released in 2 hours, measured spectrophotometrically at $285 \mathrm{~nm}$, was obtained. The results of the experimental design indicated that the three factors influence the released amount of AIA in 2 hours, where a higher concentration of chitosan increases the rate of release, while the higher concentration of TPP and longer gelation time in $\mathrm{NaOH}$ decreases the release rate.
\end{abstract}

Key word: Factorial design, controlled release, 1,3 indoleacetic acid, chitosan.

\footnotetext{
${ }^{a}$ Facultad de Ingeniería Industrial, Instituto de Investigación, Universidad de Lima. Av. Javier Prado Este 4600, Urbanización Fundo Monterrico Chico, Santiago de Surco, Lima, Perú, avalderr@ulima.edu.pe.

${ }^{\text {b }}$ Facultad de Ciencias, Escuela Profesional de Química, Universidad Nacional de Ingeniería Av. Túpac Amaru 210, Lima 25, Perú.
} 


\section{INTRODUCCIÓN}

Las hormonas vegetales (fitohormonas) son un grupo de sustancias orgánicas de origen natural, que influyen en los procesos de crecimiento, diferenciación y desarrollo de un vegetal, estas son: auxinas, giberelina, citoquininas, ácido abcísico y etileno ${ }^{1}$. La administración exógena de estas hormonas ganó relevancia a partir de los años 90; sin embargo, su uso fue limitado debido a que sus efectos biológicos tienen una estrecha región de concentración efectiva. En los últimos años, sistemas de liberación controlada de agroquímicos, en general, han recibido una gran atención. Esta tecnología tiene la ventaja de proteger de la degradación la sustancia a liberar, además de dosificar en forma adecuada a la planta, logrando mayor eficiencia en el uso de estas hormonas y por tanto un ahorro económico².

Entre los sistemas de liberación controlada resaltan las matrices biopoliméricas de quitosano, un polisacárido cuyas propiedades de biocompatibilidad, biodegradabilidad, no tóxico, y mucoadhesivo hizo que tenga un gran progreso para aplicaciones en medicina y farmacia ${ }^{3}$.

En las aplicaciones para agricultura, gran parte de la literatura se refiere al quitosano, el cual, en 1978, se propuso como membrana protectora para la liberación controlada de agroquímicos. Posteriormente, se utilizaron películas y perlas fabricadas con hidrogeles de quitosano y sus derivados para la liberación controlada de urea y atrazina, un herbicida de uso común para controlar la cizaña en el maíz. Las matrices usadas sirvieron para controlar la liberación de los agroquímicos estudiados y extender su tiempo de liberación hasta 180 veces mayores que cuando estos se aplicaban solos. En el 2001, el quitosano tuvo efectos beneficiosos en plantas florales y de cosechas. Hacia el 2007 se estudió la adsorción de paraquat con polímeros naturales, $\mathrm{y}$ al año siguiente la quitina y el quitosano mostraron algunas potencialidades para la agricultura en Latinoamérica ${ }^{4}$. En el 2011, el quitosano aparece registrado por la Agencia de Protección Ambiental Estadounidense (EPA) como bioplaguicida para cultivos de uvas y fresas. Al año siguiente, se aplicó la microencapsulación con alginato en alimentos ${ }^{5}$, y en el 2014 se probó el efecto del recubrimiento de quitosano y alginato en la liberación controlada de fertilizantes aplicados al suelo 6 .

A pesar de estos logros, aún existe un gran desconocimiento de sus aplicaciones potenciales en el encapsulamiento de ingredientes activos para la agricultura ${ }^{7}$. En la actualidad se tienen ciertas referencias del estudio de quitosano ${ }^{8,9}$ como matrices para la liberación controlada de algunos agroquímicos mientras que en el Perú, aún no se ha reportado el uso del quitosano/ alginato en la liberación controlada de hormonas vegetales.

Actualmente, varios trabajos que estudian el efecto de algunas variables que influyen en la liberación controlada, son desventajosos, debido a que utilizan muchos experimentos, no evalúan cuantitativamente el efecto que tienen estas variables y si hay algún efecto de interacción ${ }^{10}$. El Diseño Factorial de Experimentos es una herramienta estadística que nos ofrece una metodología de la experimentación; asimismo, del procedimiento para la interpretación de los resultados obtenidos, realizando menor cantidad de experimentos con la mayor fiabilidad de sus resultados. Algunos trabajos realizados en este sentido han sido 
reportados por Kincl $^{11}$ y Iurian ${ }^{12}$. Con el diseño factorial se puede hacer los estudios de los efectos de cada uno de las variables (efectos principales) y de conexión entre ellas (efectos de interacción), esta última importante debido a que muchas variables actúan en modo de interacción e influyen en la liberación controlada.

En el presente trabajo se han preparado micropartículas de quitosano cargadas con el ácido 1,3-indolacético utilizándose el diseño factorial $2^{3}$ a dos niveles para evaluar el efecto de la concentración del quitosano (biopolímero), concentración del $\mathrm{NaOH}$ (como agente gelificante) y tripolifosfato de sodio (como agente entrecruzante) en la velocidad de liberación en agua.

\section{PARTE EXPERIMENTAL}

\section{Reactivos}

El quitosano de mediano peso molecular tenía $1197 \mathrm{kDa}$ medido viscosimétricamente y $87 \%$ de grado de desacetilación medido por titulación potenciométrica con $\mathrm{NaOH}$. El ácido 1,3 indolacético (AIA) y Tripolifosfato de sodio (TPP) fueron adquiridos de SIGMA. Hidróxido de sodio y ácido acético de grado analítico de FERMONT.

\section{Equipos}

Los espectros UV fueron medidos en un espectrofotómetro modelo UV- 1800, SHIMADZU CORPORATION.

\section{Procedimiento experimental}

\section{a. Curva de calibración:}

Se disolvieron $8,8 \mathrm{mg}$ de hormona AIA en $50 \mathrm{~mL}$ de agua, luego se tomó 1 alícuota de 1 $\mathrm{mL}$ y se llevó a $25 \mathrm{~mL}$ con agua y se hizo un barrido espectral entre 190 y $400 \mathrm{~nm}$. Una vez determinada la longitud de onda de trabajo, se tomaron ciertos volúmenes para generar la curva de calibración de ALTA concentración: 2.5, 2, 1.5, 1 y $0.5 \mathrm{~mL}$ llevados a un volumen $25 \mathrm{~mL}$ con agua. Para generar la curva de calibración de BAJA concentración se tomaron los volúmenes: $0,125,250,375,500$ y $625 \mu \mathrm{L}$ llevando a un volumen final de $25 \mathrm{~mL}$ con agua.

\section{b. Ensayo de estabilidad de la hormona en medio acuoso:}

Se disolvieron 8,8 mg de hormona AIA en $50 \mathrm{~mL}$ de agua, luego se tomó 1 alícuota de $1 \mathrm{~mL}$ y se llevó a $25 \mathrm{~mL}$ con agua y se hizo un barrido espectral entre 190 y $400 \mathrm{~nm}$ durante un periodo de cuatro días. El experimento se llevó a cabo en duplicado, donde una solución fue expuesta a la luz y otra se mantuvo en la oscuridad.

\section{c. Preparación de las partículas a partir del quitosano disuelto con la hormona}

Se disolvió $1 \mathrm{~g}$ de quitosano en $50 \mathrm{~mL}$ ácido acético al $2 \%$ adicionando $0,05 \mathrm{~g}$ de hormona AIA en agitación hasta su completa disolución. Luego se dejó gotear sobre una solución de $\mathrm{NaOH} 2 \mathrm{M}$ en agitación constante a 1 y 3 horas de reposo. Las partículas se lavaron con agua abundante hasta obtener un $\mathrm{pH}$ neutro y finalmente se reticularon con tripolifosfato de sodio 
(TPP, $\mathrm{pH}=6,8)$ al $1 \%$ y al $3 \%$ por 24 horas. Se filtraron, lavaron y secaron al vacío en un desecador. Todo este proceso se llevó a cabo con los materiales cubiertos con papel aluminio para evitar el contacto con la luz.

\section{d. Experimento de liberación de la hormona}

El estudio de la liberación controlada se llevó a cabo en agua. A $25 \mathrm{~mL}$ de agua se agregaron $60 \mathrm{mg}$ de las partículas cargadas con AIA y se agitó a $130 \mathrm{rpm}$ en agitador magnético a temperatura ambiente. A distintos tiempos, se tomó $1 \mathrm{~mL}$ de la solución acuosa y se reemplazó con $1 \mathrm{~mL}$ de agua. Se obtuvo el espectro UV y se midió la absorbancia a $280 \mathrm{~nm}$.

\section{e. Diseño experimental}

Se realizó un diseño factorial 23 para evaluar el efecto de tres factores que influyen en la preparación de las partículas cargadas: concentración del quitosano (\% de quitosano), tiempo de gelación en hidróxido de sodio (tiempo) y porcentaje de tripolifosfato de sodio (\% TPP). La preparación de las partículas cargadas se realizó según el procedimiento general del apartado c, los niveles de cada uno de los factores se encuentran en la tabla 1:

Tabla 1. Valores de los factores estudiados a Nivel (-) y Nivel (+)

\begin{tabular}{|l|c|c|}
\hline Factor & Nivel (-) & Nivel (+) \\
\hline \% quitosano & 1,5 & 2,0 \\
\hline tiempo & $1 \mathrm{~h}$ & $3 \mathrm{~h}$ \\
\hline$\%$ TPP & $1 \%$ & $3 \%$ \\
\hline
\end{tabular}

Para el diseño factorial $2^{3}$ se prepararon ocho matrices de partículas cargadas para realizar ocho experimentos de liberación a los niveles indicados en la tabla 1 , y se codificaron según se muestra en la tabla 2.

A partir de cada una de estas matrices se realizaron las pruebas de liberación controlada de la AIA (tal como se indica en párrafo d) en diferentes intervalos de tiempo. La cantidad liberada en un lapso de 120 minutos se tomó como respuesta para el estudio del diseño factorial $2^{3}$. 
Tabla 2. Matriz del Diseño Experimental para la preparación de las partículas de quitosano cargadas con AIA

\begin{tabular}{|c|c|c|c|c|}
\hline No & Muestra & $\begin{array}{c}\text { X1 (\% } \\
\text { Quitosano) }\end{array}$ & $\begin{array}{c}\text { X2 (tiempo en } \\
\text { NaOH) }\end{array}$ & X3 (\% TPP) \\
\hline $\mathbf{1}$ & M1 & 1,5 & $1 \mathrm{~h}$ & 1 \\
\hline $\mathbf{2}$ & M2 & 2,0 & $1 \mathrm{~h}$ & 1 \\
\hline $\mathbf{3}$ & M3 & 1,5 & $3 \mathrm{~h}$ & 1 \\
\hline $\mathbf{4}$ & M4 & 2,0 & $3 \mathrm{~h}$ & 1 \\
\hline $\mathbf{5}$ & M5 & 1,5 & $1 \mathrm{~h}$ & 3 \\
\hline $\mathbf{6}$ & M6 & 2 & $1 \mathrm{~h}$ & 3 \\
\hline $\mathbf{7}$ & M7 & 1,5 & $3 \mathrm{~h}$ & 3 \\
\hline $\mathbf{8}$ & M8 & 2,0 & $3 \mathrm{~h}$ & 3 \\
\hline
\end{tabular}

\section{RESULTADOS Y DISCUSIÓN}

\section{a. Curva de calibración:}

La figura 1 (a) muestra el espectro UV de la hormona (AIA) disuelta en agua donde se puede apreciar una banda de mayor absorbancia en $\lambda_{1}=228.7 \mathrm{~nm}$ correspondiente a las transiciones $\mathrm{n} \rightarrow \pi$ del grupo carboxílico y la segunda de menor absorbancia a $\lambda_{2}=280.9 \mathrm{~nm}$ correspondiente a las transiciones $\pi \rightarrow \pi$ de los dobles enlaces alternados del grupo indol.

A la misma concentración, pero en este caso disuelta en ácido acético $2 \%$, el espectro de la hormona muestra una sola banda (figura 1 (b)) en $\lambda_{2}=280.9 \mathrm{~nm}$ correspondiente al grupo indol. En este espectro ya no se observa la banda que corresponde a las transiciones del grupo carboxílico, por lo que se ha determinado a $\lambda_{2}=280.9 \mathrm{~nm}$ como la longitud de onda de referencia para los ensayos de calibración y de liberación controlada de la hormona.

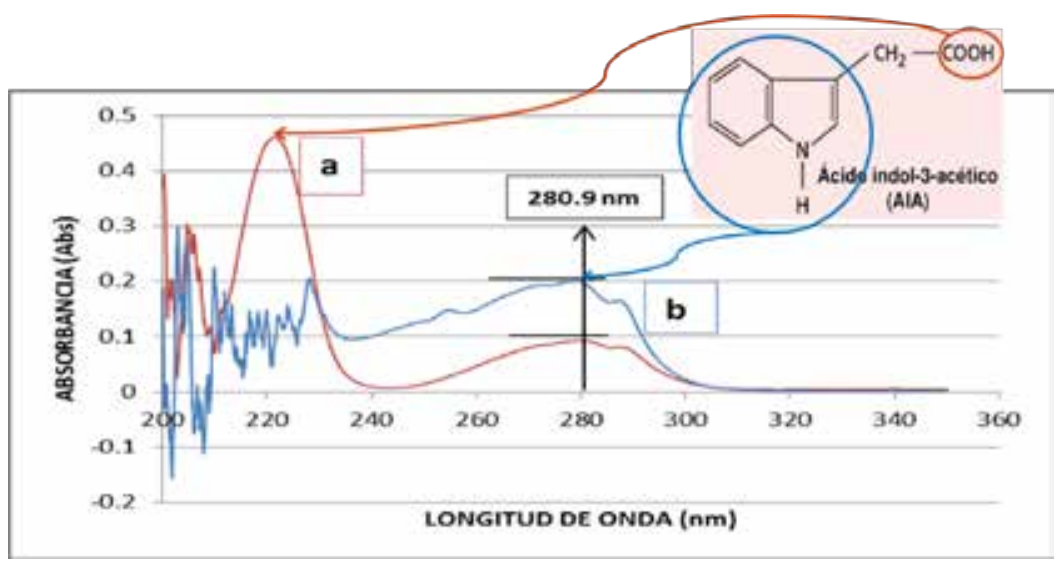

Figura 1. Espectro UV de la hormona ácido 1,3-indolacético (AIA) en medio (a) acuoso y (b) ácido acético $2 \%$ 
Una vez determinada la longitud de onda máxima se obtienen las curvas de calibración de alta y baja concentración como se muestra en la figura 2 ( $a$ y b ), respectivamente.
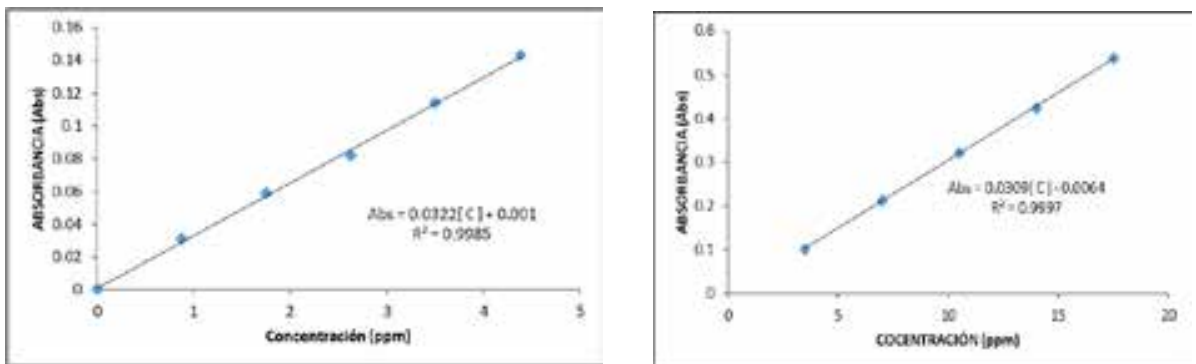

Figura 2. Curva de calibración de la hormona AIA: (a) baja y (b) alta concentración.

\section{b. Ensayo de estabilidad de la hormona en medio acuoso:}

En la figura 3 se puede evidenciar que cuando la hormona es expuesta a la luz, la absorbancia de sus bandas va disminuyendo, es decir disminuye su concentración mientras el perfil de su espectro se mantuvo semejante. La literatura señala que la hormona en solución sufre un proceso de foto-oxidación y se transforma en compuestos biológicamente inactivos de naturaleza lactónica. La saturación del doble enlace en el anillo produce la pérdida de la actividad hormonal.

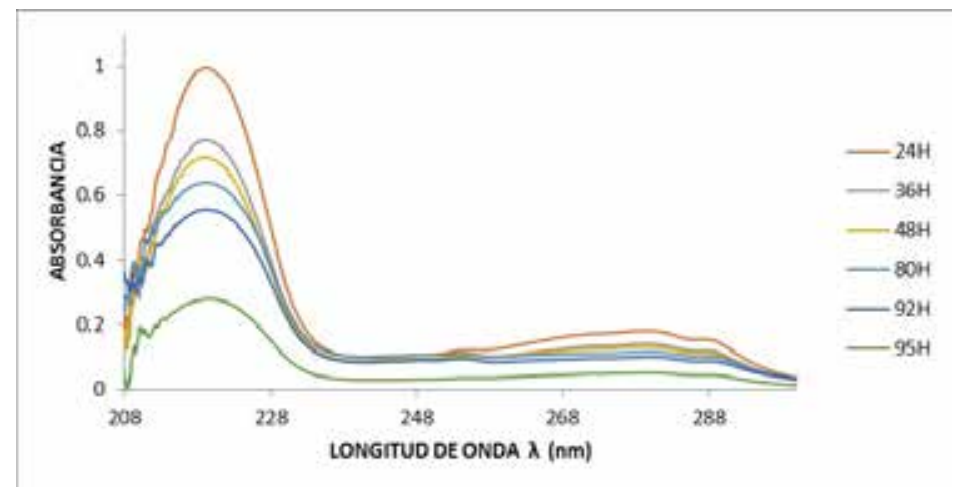

Figura 3. Espectros UV de la hormona (AIA) expuesta a la luz en diferentes tiempos. 
Cuando se repite el procedimiento, pero en la oscuridad, se obtuvo la figura 4, donde se puede observar la integridad de la hormona en aproximadamente el mismo tiempo de experimento.

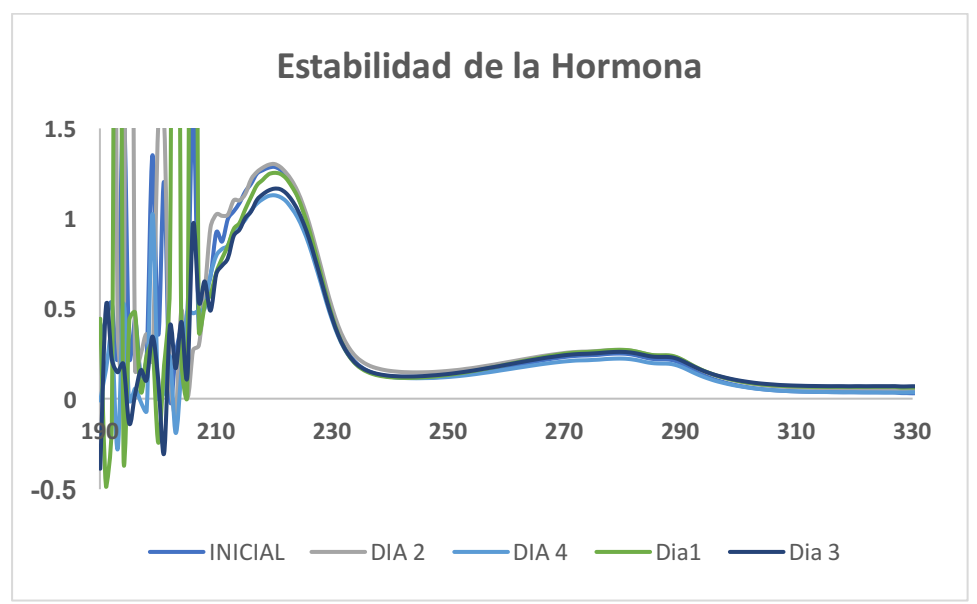

Figura 4. Espectros UV de soluciones acuosas de la hormona AIA en la oscuridad

Los resultados de este análisis conllevaron a realizar los ensayos de la liberación, cubriendo con papel aluminio todo el material así como el uso de material color ámbar en la recepción de las muestras de la liberación de la hormona.

\section{c. Efecto de factores con el Diseño Factorial}

El porcentaje de AIA liberada de todas las partículas preparadas según la tabla 2 se encuentra graficado en las figuras $5 \mathrm{a}$ y $5 \mathrm{~b}$, las cuales muestran las diferencias en la velocidad de liberación según el método de preparación de las partículas de quitosano cargadas con la hormona.

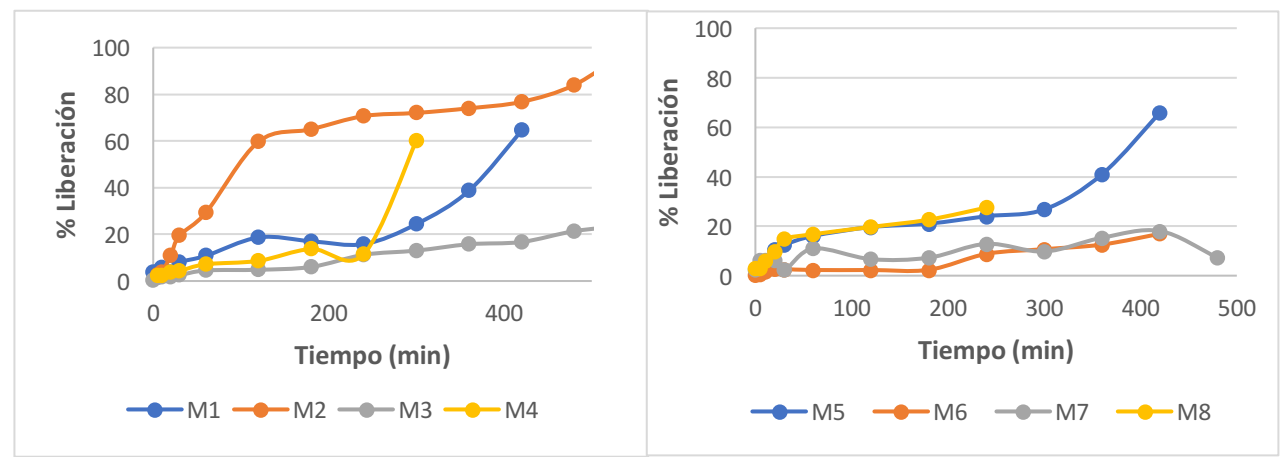

Figura 5. Perfil de liberación del ácido 1,3-indolacético (AIA) en: a) TPP $1 \%$ y b) TPP $3 \%$. 
Se observa, en general, que en TPP al 3\% hay una menor liberación, esto debido a que probablemente a estas concentraciones el TPP forma un mayor enrejado que controla la velocidad de liberación.

Para estimar los efectos que presentan los factores estudiados en la liberación, se hizo un análisis del diseño factorial utilizando el software MINITAB 16 tomando como respuesta la cantidad liberada a $120 \mathrm{~min}$. Los resultados se muestran en la figura 6.

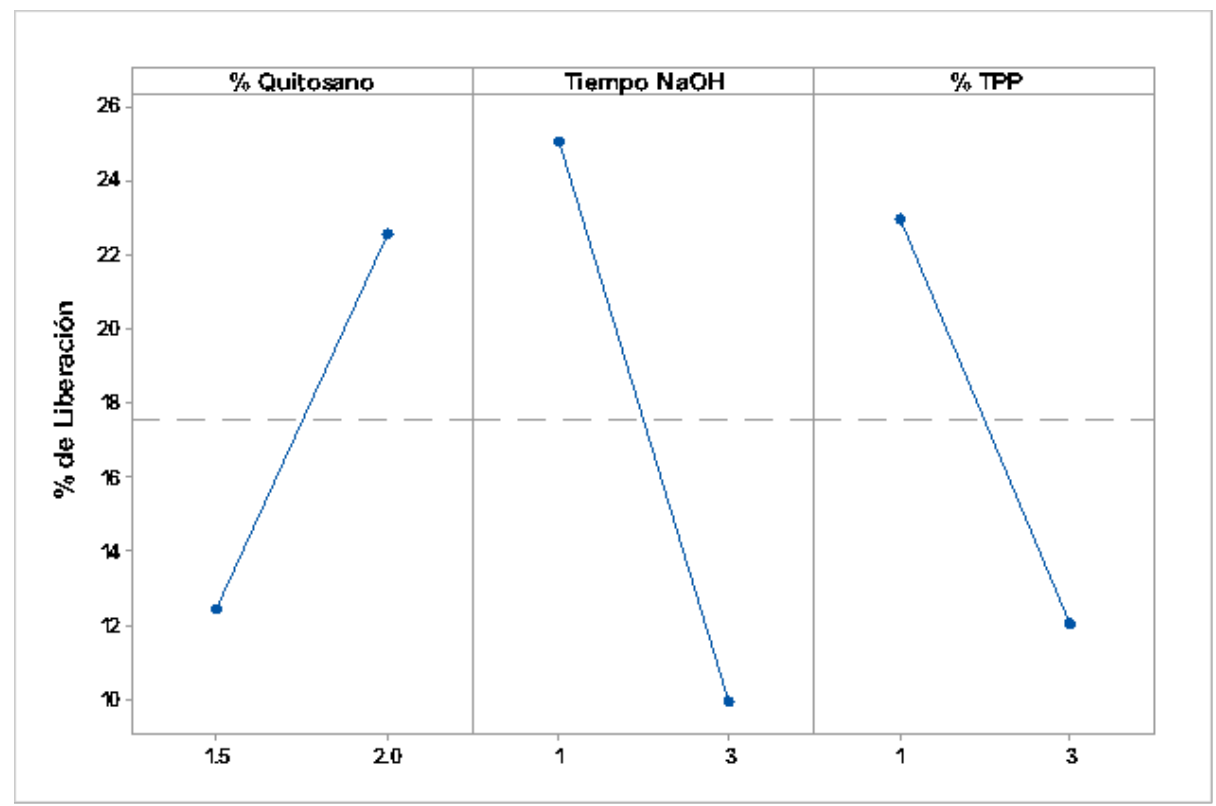

Figura 6. Gráfica de los efectos del \% quitosano, tiempo de gelación en $\mathrm{NaOH}$ y \% de TPP en la cantidad liberada a $120 \mathrm{~min}$.

Los resultados muestran que los tres factores influyen en la cantidad del AIA liberada de las partículas de quitosano a las 2 horas de ensayo. Tanto el tiempo de gelación del quitosano en $\mathrm{NaOH}$ y el \% de TPP disminuyen el \% de liberación a niveles altos, esto debido a que dichos factores influyen en la formación del entrecruzamiento del gel; por otro lado, el aumento en la concentración del quitosano aumenta el \% de liberación del AIA, quizás porque este aumento de la concentración favorece el hinchamiento de la partícula en el proceso de liberación, favoreciendo la liberación del AIA.

Evaluando también los resultados de los efectos de interacción de los factores estudiados, estos se encuentran en la figura 7 y se llevaron a cabo con MINITAB 16. 


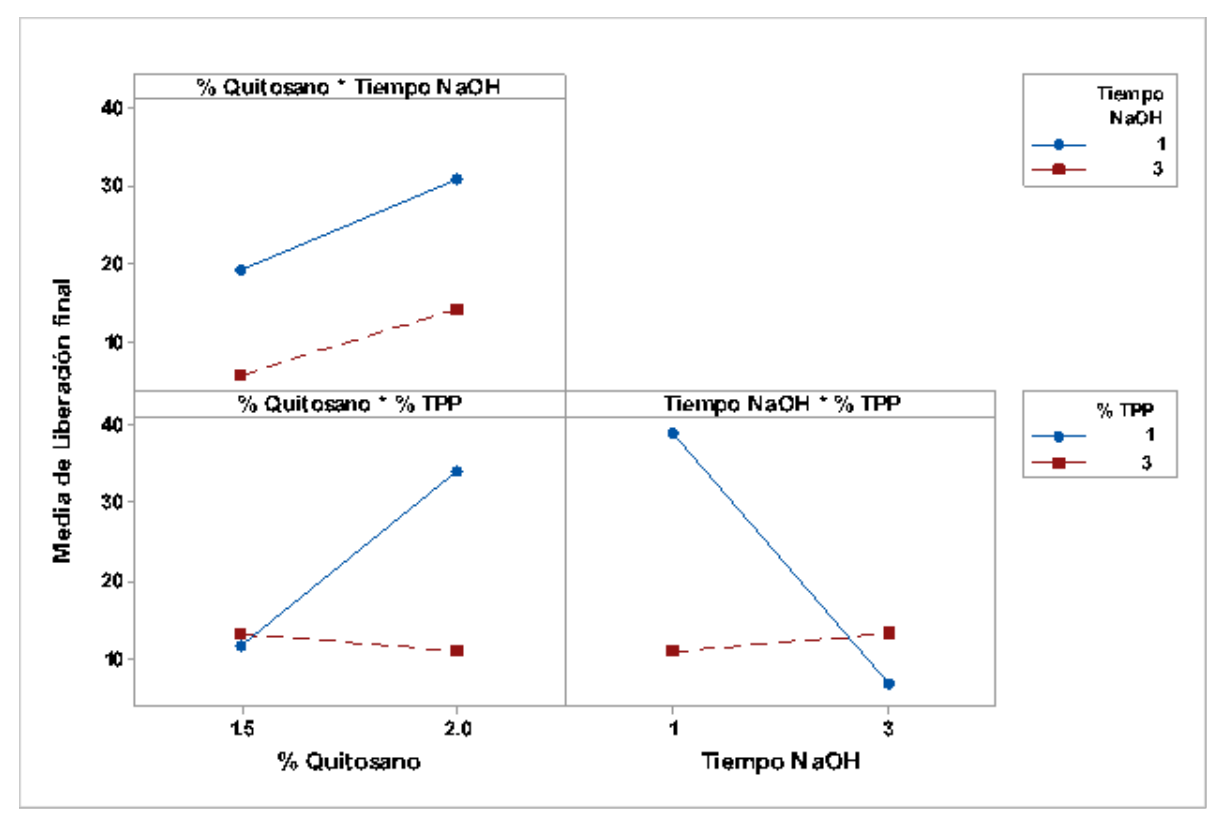

Figura 7. Gráfica de los efectos de interacción del \% de quitosano, tiempo de gelación de $\mathrm{NaOH}$ y \% TPP.

La figura 7 muestra que existen efectos de interacción entre los parámetros medidos, esto es, el efecto que originan el \% de quitosano y el tiempo de gelación en $\mathrm{NaOH}$ sobre el \% de liberación del AIA, se ve influenciado según el nivel en el que se encuentra del \% de TPP, y esto debido a que quizás aquí tengan que ver efectos combinados en el entrecruzamiento con el grado de hinchamiento de las partículas preparadas. El estudio de todos estos efectos nos permite evaluar las condiciones necesarias para una adecuada velocidad de liberación del AIA en medio acuoso.

Con los resultados obtenidos se pudo también obtener una gráfica de superficie de la liberación del AIA en función de los dos factores que más influyen en la liberación del AIA, es decir del $\%$ de quitosano y \% de TPP a nivel de tiempo de $\mathrm{NaOH}$ de 2 horas, estos resultados se muestran en las figuras 8 y 9 . 


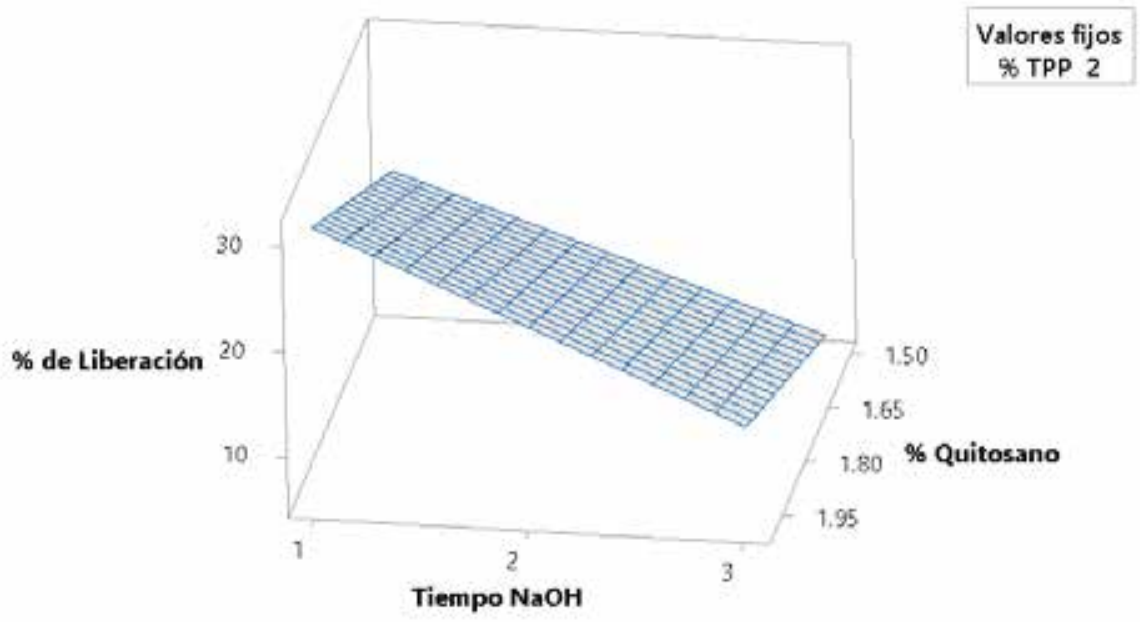

Figura 8. Gráfica de superficie del \% de quitosano y tiempo de gelación en $\mathrm{NaOH}$ en la liberación del AIA de la partícula de quitosano.

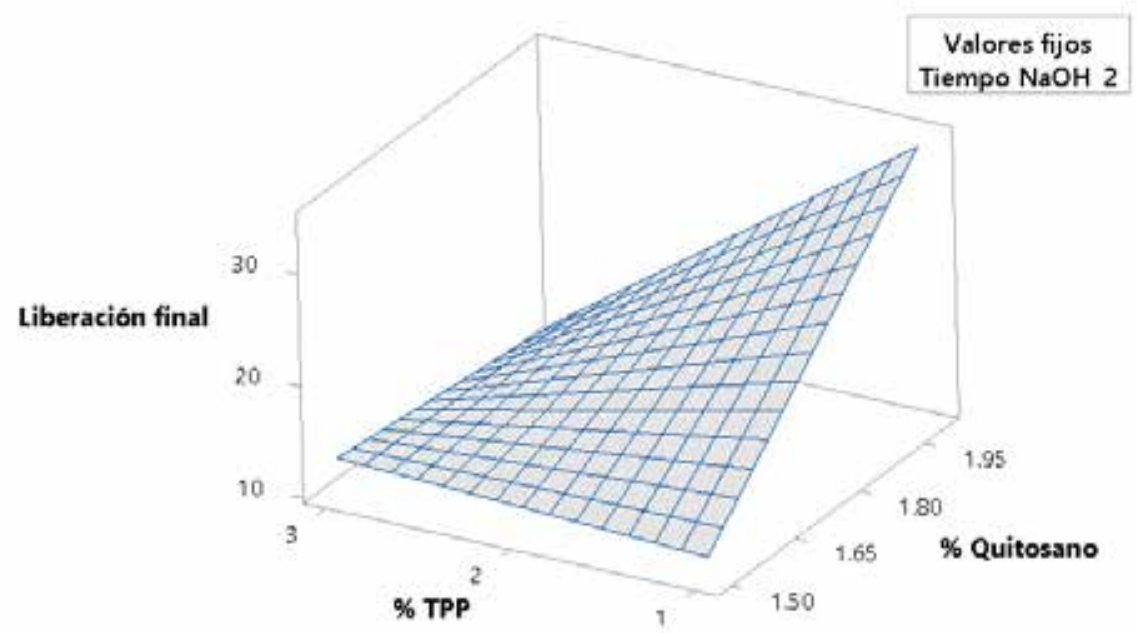

Figura 9. Gráfica de superficie del \% de quitosano y \% de TPP en la liberación del AIA de la partícula de quitosano. 


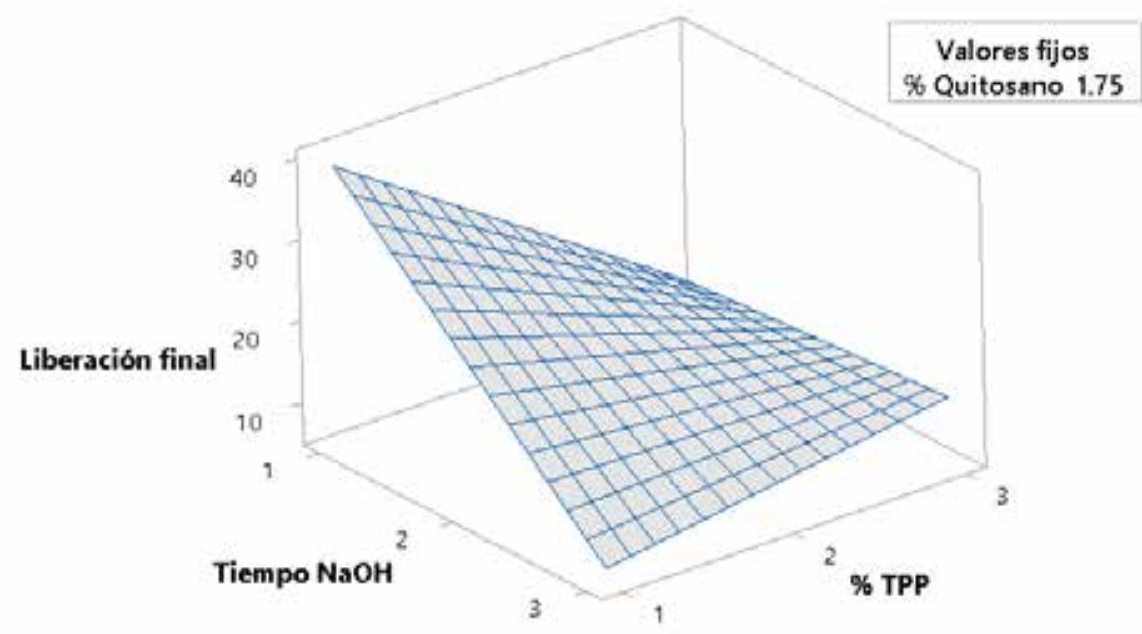

Figura 10. Gráfica de superficie del \% de TPP y tiempo de gelación en $\mathrm{NaOH}$ en la liberación del AIA de la partícula de quitosano.

La gráfica de la figura 10 nos muestra que es posible realizar un modelo matemático de predicción de liberación del AIA en medio acuoso a partir del \% de quitosano, tiempo de gelación en $\mathrm{NaOH}$ y \% de TPP.

\section{CONCLUSIONES}

La aplicación del Diseño Factorial $2^{3}$, ha demostrado ser un método estadístico viable para ser aplicado en ensayos donde son varios los parámetros que influyen en los procesos y que hay que evaluar.

Gracias a este diseño experimental podemos proponer ensayos más certeros y optimizar la matriz de quitosano para la liberación controlada de la hormona AIA.

El diseño experimental aplicado en este trabajo, indica que los tres factores estudiados influyen en la cantidad liberada de AIA en un periodo de 2 horas, en donde a mayor concentración de quitosano aumenta la velocidad de liberación, mientras que a mayor concentración de TPP y mayor tiempo de gelación en $\mathrm{NaOH}$ disminuye la velocidad de liberación.

\section{AGRADECIMIENTO}

Agradecemos al Instituto de Investigación de la Universidad de Lima (IDIC), por los aportes económicos para el desarrollo de este tema de investigación. 


\section{BIBLIOGRAFÍA}

1. Lluna Duval R. Hormonas vegetales: crecimiento y desarrollo de la planta. Ind Hortícola. 2006;196:22-6.

2. Tsatsakis AM, Shtilman MI. Polymeric derivatives of plant growth regulators: synthesis and properties. Plant Growth Regul. 1994;14(1):69-77.

3. Fan L, Jin R, Le X, Zhou X, Chen S, Liu H, et al. Chitosan microspheres for controlled delivery of auxins as agrochemicals. Microchim Acta. 2012;176(3-4):381-7.

4. Lebrón O. R. Projecte fi de carrera. 2007.

5. Lupo B, González C, Maestro A. Microencapsulación con alginato en alimentos. Técnicas y aplicaciones. Rev Venez Cienc y Tecnol Aliment. 2012;3(1):130-51.

6. Aguilar Y. Preparación y evaluación en suelo de fertilizantes de liberación controlada ( NPK ) cubiertos con polímeros biodegradables. 2014;

7. Kashyap PL, Xiang X, Heiden P. Chitosan nanoparticle based delivery systems for sustainable agriculture. Int J Biol Macromol [Internet]. 2015;77:36-51. Available from: http://dx.doi.org/10.1016/j.ijbiomac.2015.02.039

8. Szopko R, Schmidt C, Peniche C. Novel drug delivery systems : Chitosan conjugates covalently attached to steroids with potential anticancer and agrochemical activity Javier Pérez Qui nones. 2009;84(2011):858-64.

9. Wu L. Preparation and properties of chitosan-coated NPK compound fertilizer with controlled-release and water-retention. 2008;72:240-7.

10. Fan L, Jin R, Le X, Zhou X. Chitosan microspheres for controlled delivery of auxins as agrochemicals. (2012):381-7.

11. Kincl M, Turk S, Vrečer F. Application of experimental design methodology in development and optimization of drug release method. Int J Pharm. 2005;291(1-2):3949.

12. Iurian S, Turdean L, Tomuta I. Risk assessment and experimental design in the development of a prolonged release drug delivery system with paliperidone. Drug Des Devel Ther [Internet]. 2017;11:733-46. Available from: https://www. scopus.com/inward/record.uri?eid=2-s2.0-85015324888\&doi=10.2147\%2FDDDT. $\mathrm{S} 125323 \&$ partnerID $=40 \& \mathrm{md} 5=795 \mathrm{db} 3 \mathrm{a} 441 \mathrm{fc} 81 \mathrm{c} 6 \mathrm{f} 259 \mathrm{f} 46 \mathrm{a} 8 \mathrm{fadb} 4 \mathrm{c} 2$ 